\title{
Continuum Modelling and Representations of Interfaces and their Transitions in Materials
}

\author{
Catherine M. Bishop ${ }^{\mathrm{a}, \mathrm{b}, *}$ Ming Tang ${ }^{\mathrm{b}}$ Rowland M. Cannon ${ }^{\mathrm{c}}$ \\ W. Craig Carter ${ }^{b}$ \\ ${ }^{a}$ Department of Materials, Oxford University, Oxford, OX1 3PH UK \\ ${ }^{\mathrm{b}}$ Department of Materials Science and Engineering, Massachusetts Institute of \\ Technology, Cambridge, MA 02193, USA \\ ${ }^{\mathrm{c}}$ Materials Sciences Division, Lawrence Berkeley National Laboratory, Berkeley, \\ CA 94720, USA
}

\begin{abstract}
Evidence for internal and external interface transitions in ceramics and metals is copious. The work described here lays out a framework for predicting interface transitions and including them on bulk phase diagrams. Advances in understanding possible grain boundary transitions in single- and binary-component materials have been made with energetics modelled with the KWC phase-field model. Analyses inspired by Cahn's critical point wetting work allowed the exploration of the stable, metastable and unstable grain boundary compositions and structures. A general phase-field model has also been developed that allows the couplings between chemistry, structure, and electrostatics to be explored. This model has been applied to grain boundaries in silicon nitride and indicates that intergranular films can be stabilized below the eutectic temperature. Predictive information from the models presented here could be used to inform material processing routes in order to design
\end{abstract}


for interface structures and so bulk properties.

Key words: phase-field model and grain boundary

wetting and thermodynamics and ceramics and interface structure

\section{Introduction}

Surfaces and interfaces play important roles in material behavior. While surfaces and interfaces share many continuum aspects - each being two dimensional defects that must appear in any finite, or polycrystalline, or multi-phase material - interfaces have much greater complexity and are much more difficult to study experimentally. The increased complexity has many sources including structural changes, crystallographic complexity, stresses, and charged defects. Atomistic calculations successfully reproduce ground-state structures and energies, but only for a limited number of relatively special interface inclinations, misorientations, and chemistries. However, predictions of interfacial behavior in materials that will be used or fabricated in open environments at high temperatures will be necessary.

This is important because at equilibrium, interfaces can exhibit changes in configuration, composition, and resultant properties with changes in temperature or solute chemical potential; these often occur continuously, but may entail discontinuities. The structure changes at such transitions are often accom-

* University of Oxford, Department of Materials, Parks Road, Oxford, OX1 3PH, UK. Phone +44 (0)1865 273672. Fax +44 (0)1865 283333

Email address: catherine.bishop@materials.ox.ac.uk (Catherine M. Bishop). 
panied by very large changes in associated kinetic, physical, and mechanical properties. In addition, it is possible, especially in some ceramics, to retain the interfacial structures and compositions from high temperature with suitable cooling. The implication is that it becomes fruitful to consider using heat treatment at specific temperatures to adjust an interface to give desired properties at room temperature, if the interfacial structure-stability relations are known. Thus, understanding these issues provides rich opportunities for technological exploitation.

The concept of transitions for interfaces in or on solids extends back almost a century. Since it was established that the atomic structure of surfaces could change in response to the driving force during crystal growth or from thermally induced roughening $[1,2]$, various notions of structure or complexion transitions for solid surfaces have gained vitality, specificity and refinement, both from theory and experiment [3-5]. Examples include: transitions in the reconstructions of the pure solid surfaces $[3,5]$, thermally induced surface roughening $[4,6]$ and then pre-melting [4,5,7-10]; for adsorbate overlayers on unchanged substrates, clustering [11] and various other ordered or disordered configuration changes [12-15], monolayer to multilayer adsorption and then wetting transitions $[16,17]$, and adsorption induced surface roughening [16]; finally, defaceting associated with roughening transitions [18] which may or not involve adsorption [19]. Affirmation and refinement has been possible owing to a suite of quantitative probes of surface structure and composition involving diffraction of electrons or x-rays (from synchrotrons), ion scattering, atom probes, and various surface sensitive spectroscopies.

Of particular interest in terms of thermodynamic surface property transitions is Cahn's critical point wetting theory [20] for which key predictions were 
soon affirmed [21]. This describes the existence of a wetting transition temperature above which one of two immiscible liquids (with the lower surface energy) wets the other, and moreover, associated with this is a transition condition in the single phase regime beyond which an atomically thick, but graded surface adsorption layer forms, enriched in the atoms giving lower surface energy. The predicted conditions for continuous and discontinuous changes in the equilibrium surface segregation types were also plotted onto a bulk phase diagram. To our knowledge, this was the first example of the inclusion of the surface, as a necessary defect, onto a phase diagram that could be utilized by designers of material processes. Moreover, the equilibrium thermodynamic state of the surface, including its characteristic thickness and composition, could be predicted, plotted, and utilized to design processes to produce stable microstructural features. In effect, the inclusion of an adsorption transition condition and critical wetting point onto the temperature-composition axis produced a "surficial-defect phase diagram" (see Fig. 1.)

This surficial phase diagram follows from a continuum thermodynamic model of the surface and, therefore, does not include information such as structure, site-specific adsorption or orientation dependence. Since then, however, many more examples have emerged in which two dimensional phase diagrams have been developed to describe different regimes of atomic configurations of an adsorbate overlayer on an invariant surface, e.g., see [13-15], or to depict adsorption and wetting transitions from lattice gas or similar models $[16,17]$, that can include various refinements from long range forces or from mixing of substrate and adsorbate atoms [22]. It is a plausible goal, then, that some such microscopic information could be incorporated into continuum models that can treat both external surfaces and internal interfaces, the latter being 
the focus of this paper. If sufficiently accurate, such models have the advantage that they can not only describe equilibrium interfacial complexions and their transitions over the broad range of temperature, pressure, and composition space, but that they can also be readily used to provide quantitative models for kinetic and dynamic behaviors in chemically heterogeneous systems during processes such as phase transformations, grain coarsening or fluid spreading.

In parallel, analogous behavior for grain boundaries or other internal interfaces may be expected. However, this problem is conceptually, physically and experimentally more complex; thus, affirmation of ideas and theories has been more limited or indirect. There have been thermodynamic arguments for the existence of and conditions for structure transitions at interfaces [23-25]. Simplified models for structural transitions in grain boundaries have been developed, but often these being model specific serve to stimulate the imagination while having limited connection to specific materials, e.g., [26-28]. Investigation by transmission electron microscopy (TEM) of grain boundaries in some pure metals indicates that preferential melting of grain boundaries does occur, but there is no significant thickening of the core by pre-melting until within $1^{\circ} \mathrm{C}$ of bulk melting $\left(0.999 T_{m}\right)[29,30]$. There have been observations of faceting/defaceting transitions, initiating between $0.5-0.9$ of $T_{m}$, but often these reflect impurity influences [31,32]. More generally, for multicomponent systems, however, several types of observations illustrate that the situation is more interesting. Multilayer grain boundary adsorption has been seen in several metallic systems [33]. One important case is that of $\mathrm{Bi}$ doped $\mathrm{Cu}$, in which systematic transitions between monolayer to multilayer adsorption with increasing chemical potential of $\mathrm{Bi}$ and a complex temperature dependence have been documented and modeled [34-36]. 
In addition, in metallic systems, provocative macroscopic observations include changes at certain temperatures in dihedral angles or interfacial tensions, interface penetration, and unexpectedly strong temperature or composition dependencies for grain boundary diffusion [25,37-40]. Observations from grain growth that migrating boundaries in metals can contain non-equilibrium adsorption levels or can exhibit abrupt changes in kinetics at some specific temperature have motivated proposals for structural changes [41-43], although some of this may confuse kinetic and thermodynamic effects. Also, there has been a continually accumulating body of information involving impurity activated grain growth [44-47] or "activated" sintering for metals and ceramics $[48,49]$. Such behavior can be reminiscent of liquid phase effects but often occurs below the relevant eutectic temperature or impurity level for bulk liquid to be stable, implying that the rapid kinetics ensue from boundary structure changes.

In general, such changes have not been well related to quantitative changes in boundary composition or structure. However, there has been a body of microscopical study of wide, disordered or sometimes wetted grain boundaries in various ceramics in the presence of glass forming (often silicate based) impurities, as has been reviewed elsewhere [50-53]. In $\mathrm{Si}_{3} \mathrm{~N}_{4}$ [54-56], $\mathrm{SiC}$ [57-59], Pb-ruthenate/Pb-Al-silicate [60], $\mathrm{Al}_{2} \mathrm{O}_{3}$ [61,62], or $\mathrm{ZnO}$ with $\mathrm{Bi}_{2} \mathrm{O}_{3}$ [63-65], the grain boundaries can be 1-2nm wide, disordered layers. In some instances, such as with $\mathrm{Al}_{2} \mathrm{O}_{3}[61,62]$ or $\mathrm{MgO}[66,67]$, the grain boundaries in polycrystals can be penetrated by silicate liquid, indicating equilibrium or transient wetting occurs $[51,52]$. These situations have been extensively investigated by high resolution and analytical TEM (in part, as the structures are retained during cooling from high temperature.) In two systems, specifically several different 
$\mathrm{Si}_{3} \mathrm{~N}_{4}$-based ones [54-56,68-71,52], and Pb-ruthenate in a $\mathrm{Pb}-\mathrm{Al}$ silicate [60], it has been shown unambiguously that the average boundary compositions differ from that of the bulk liquid (retained as glass on cooling), the thicknesses depend on the chemical potentials of additives in the system, and in the presence of large excesses of liquid, the boundary films remain finite (rather than infinite) in thickness. This means these interfaces can be considered as multilayer, adsorbate films in equilibrium with the bulk liquid, which itself is not fully wetting. Moreover, in another instance, it has been shown that a change in the boundary structure in $\mathrm{ZnO}$ doped with $\mathrm{Bi}_{2} \mathrm{O}_{3}$ occurs leading to a similar nanometer wide boundary forming below the eutectic that causes the activated sintering [49]. And in yet another, a similarly observed structural transition occurs sub-solidus and has been associated with rapid abnormal grain growth in alumina [47].

Thus, there are needs to relate the detailed behavior of specific boundaries to the structure, and parallel needs to provide a general framework to describe the existence conditions of various boundary complexions and complexion transitions in the context of the bulk phase diagrams. The Cahn critical point wetting model [20] is a useful starting point, as has been appreciated by others in application to multicomponent solid/liquid systems [72,73]. The proposed existence regimes can be mapped onto phase diagrams showing the effects of changing thermodynamic variables. A reasonable extension is shown in Fig. 1(b) for a system forming a simple eutectic with attention to the side with the more refractory solid, A. In the spirit of critical point wetting theory, once a boundary begins to disorder, it might be anticipated that for higher temperatures at solid/liquid co-existence the liquid should inevitably be wetting. However, as mentioned above, evidence exists that in certain systems 
significant boundary disorder can occur well below the eutectic and yet the liquid may not be fully wetting along boundaries until achieving a transition temperature that is well above the eutectic. Such is shown schematically in Fig. 1(b). In $\mathrm{ZnO}$ containing small amounts of $\mathrm{Bi}_{2} \mathrm{O}_{3}$, several aspects of this behavior have been observed in that nanometer wide disordered boundaries exist over a wide temperature range that spans the eutectic. This range is bounded by a wetting transition above which the liquid films at the grain boundary become arbitrarily thick and the dihedral angles go to zero; this has been found to occur well above the eutectic [63-65,74]. In addition, disordered boundaries have been seen to form stably below the eutectic [49].

We present a review of results from a theoretical analysis [75,76] and from numerical calculations using an expanded model and based on the Si-O-N phase diagram $[77,78]$. The concepts that derive from this interfacial wetting theory are previewed in Fig. 1(b): a type of expected behavior for the prototypical eutectic phase diagram. The single-phase wetting curve that extends from the $A$-rich two-phase liquid-solid region corresponds to the wetting curve in Fig. 1(a), except that the two phases do not have a consulate point. The absence of a consulate point and the need to describe a structural transition as well require extension to Cahn's original analysis, but similar behavior is anticipated except that two transitions rather than one can be expected. In Fig. 1(b), the multilayer (or drying) transition temperature $T_{M L}$ separates the two-solid phase region into two regimes: for $T<T_{M L}$, grain boundary interfaces for the $A$-rich phase will have site-specific (Langmuir) absorption of a limited amount of $B$-component; at $T>T_{M L}$, segregation of $B$ to grain boundaries of the $A$-rich phase is multilayer. The lower transition curve also illustrates that analogous thickness and composition transitions occur in single 
phase polycrystalline materials.

High adsorption at tilt grain boundaries modelled by a two-dimensional lattice gas was found by Kikuchi and Cahn [27], giving a result which was prescient, if from a highly simple model. At low temperatures, solute adsorbs at the boundary at low enthalpy sites, similarly to the widely seen Langmuir-McLean type behavior. This adsorption decreases with temperature as entropic effects become more important. At temperatures approaching the melting point, adsorption of the minority component again increases continuously as the boundary pre-melts. These trends are illustrated in Fig. 2(a). In real grain boundaries, it is possible that distinct multilayer adsorption and wetting transitions could occur. It is also possible that these could entail first-order rather than continuous transitions. These possibilities are illustrated in Fig. 2(b). If there are first order transitions from monolayer to multilayer adsorption (as indicated by the dashed lines in Fig. 2(b)), then we would expect a $T_{M L}$ and line on the corresponding phase diagram, Fig. 1(b).

In the schematic, Fig. 1(b), $T_{M L}$ is approximated as a characteristic temperature with a value at coexistence considerably lower than the eutectic temperature. The grain boundary pre-melting temperature would be expected to be considerably reduced in materials where the entropy change upon devitrification is small, such as in materials in which significant fixed short-range order exists in the liquid state, such as silicates. However, it is difficult to generalize macroscopic interfacial behavior from specific discrete simulations; furthermore, there are system size and simulation time constraints on atomistic calculations that frequently interfere with physical interpretation.

The single-phase grain boundary behavior curve that emanates from the two- 
phase region at $T=T_{M L}$ in Fig. 1(b) suggests two different kinds of grain boundary behavior: thick, relatively unstructured grain boundaries, with enriched compositions at $B$-rich average compositions, and thin, ordered boundaries at $A$-rich average compositions. Presumably, if equilibrium grain boundary structural thicknesses and compositions could be plotted on a diagram such as Fig. 1(b), they could be used to rationalize grain boundary behavior for engineering materials and to guide experimental determination of grain boundary film compositions.

In this paper, we review progress towards creating continuum thermodynamic models for interfaces that will lead to general theoretical predictions of interfacial phase diagrams and numerical models that will predict grain boundary compositions and widths from thermodynamic data. The simplest versions of the model account for structure and misorientation and predict both continuous and discontinuous structural changes as a function of temperature and misorientation. More complex models must include grain boundary segregation of solutes and charged defects. While these models do not provide as much detailed information about interfaces, they do provide mechanisms to characterize general behavior.

A major challenge in making these continuum models predictive is the incorporation of bulk and interface data for real materials. There is a natural way to include bulk thermodynamic data for equilibrium phases, but it is necessary to be able to calculate energy densities for non-bulk compositions and structures. Information from interfaces in specific materials is also available from atomistic and first principles calculations or atomistic characterization. In this paper, we will discuss ways of incorporating bulk and local data from experiment and simulation into continuum models of real materials. 
In classical thermodynamics, bulk equilibrium is calculated by minimizing the appropriate thermodynamic potential (for example, the Gibbs free energy at fixed temperature, pressure and composition) while excluding the energetic contribution from interfaces and defects. The effects of interfaces between phases in equilibrium with each other can be accounted for in terms of the associated extra energy or chemical content projected onto a plane of negligible volume. In diffuse-interface theory, in contrast, boundaries are no longer as if sharp but are recognized to be spread out in space and typically contain regions of composition or structure that are not stable in the bulk for the given thermodynamic conditions.

Diffuse-interface theory has been used in successful studies on for example, spinodal decomposition [79] and dendritic growth of solid from a melt [80]. The energy of a system is the integral of the energy density which has contributions from the local value of the fields and from the local spatial variation of fields. Therefore, the required interfaces must trade off their energetic contribution by smoothing out the necessary transitions in their values while keeping to a minimum the regions in space where the fields have non-bulk equilibrium values. Recent theoretical advances permit the inclusion of crystallographic fields that can characterize the misorientation and structural order of a grain boundary [81]. Although, bulk thermodynamic data can be used for modelling real systems, there are challenges associated with fitting the empirical constants and functions to available data which are discussed below.

Our recent work on grain boundary films stems from Cahn's critical wetting theory [20] and incorporates ideas treating the crystallographic phase field [81] and from interfacial absorption theory and provides a framework for discussion of phenomena of equilibrium grain boundary films. We review a two-field 
phase-field model for grain boundaries in single-component systems and the analysis of the energy relations approaching the melting point. Some results, such as a considerable decrease in the grain boundary melting temperature compared to that observed in metals, may spark controversy, but can be rationalized and should prompt new experiments. Additionally, a phase-field model for grain boundaries in heterogeneous ceramic materials will be introduced and issues relating to modelling a specific material system including the incorporation of experimental and computational data will be discussed. The details of our work are being prepared for publication elsewhere so this review will focus instead on the methods and concepts employed.

\section{Review of Critical Point Wetting in Binary Liquid System}

Cahn recognized that container walls are necessary constituents in liquid systems. He analyzed the energetics and transitions in binary liquid systems in his seminal paper on critical point wetting [20]. Here we review that paper.

In Cahn's analysis, the excess free energy of a heterogeneous liquid with local composition $c(x)$ in contact with a wall at $x=0$ and extending to infinity is postulated as

$$
\Delta F=\Phi\left(c_{s}\right)+\int_{0}^{\infty}\left[\Delta f+\kappa(d c / d x)^{2}\right] d x
$$

where $\Phi\left(c_{s}\right)$ is the interaction energy of the fluid at composition $c_{s}$ with the wall, $\Delta f$ is the excess homogeneous energy of the fluid with a double well form, and $\kappa$ is the gradient energy coefficient. The function $\Phi\left(c_{s}\right)$ would be the surface energy for a homogeneous material with no composition change 
near the surface (and no long range interaction with the container.) Using the equilibrium expressions from variational calculus and the boundary conditions, a form for the energy extrema or the equilibrium surface energy, $\sigma$, and an equation giving the composition at the wall are derived:

$$
\begin{aligned}
& \sigma=\Phi\left(c_{0}\right)+\int_{c_{0}}^{c_{s}}(\phi+2 \sqrt{\kappa \Delta f}) d c \\
& \phi= \pm 2 \sqrt{\kappa \Delta f\left(c_{s}\right)}
\end{aligned}
$$

The composition of the reservoir is $c_{0}$ and $\phi=\frac{d \Phi}{d c_{s}}$. These lead to a graphical construction when $c_{0}=c_{\alpha}$ and the $\alpha$-phase is saturated in B. The intersections of the two curves in Fig. 3(a) give equilibrium values of $c_{s}$. The area between the curves is the reduction in surface energy when the surface is at $c_{s}$ rather than $c_{0}$, the composition of the bulk fluid. As temperature changes, the shape of the $2 \sqrt{\kappa \Delta f}$ curve will change so that there can be 1 or 3 intersections of the two curves. At low temperatures, the three intersections correspond to stable, unstable and metastable surface compositions. At temperatures just below coexistence, Fig. 3(b), there is one intersection and this corresponds to perfect wetting. At a particular temperature, $T_{w}$ there will be a first-order surface transition from low adsorption to complete wetting. When the bulk equilibrium phase is undersaturated in $\mathrm{B}, 0 \leq c_{0}<c_{\alpha}$, a similar construction predicts a series of first order transitions between low and high adsorption that terminate in a surface critical point at $T_{c}$ that may be above or below the bulk critical point.

Cahn analyzed the thickness of the surface layer and found that below the first order transition, a finite but small layer of positive adsorption is stable. Above the transition line, the thickness of the adsorbed layer diverges logarithmically 
as $\left(c_{\alpha}-c_{0}\right) \rightarrow 0$.

Figure 1(a) shows all of this information plotted on the bulk phase diagram. One goal of the new research described in this paper is to advance the field towards these kinds of phase-diagrams. In addition to bulk phase equilibria, they will contain information about interface states and the transitions between those states.

A physical interpretation of this behavior can be achieved by considering the situation above the wetting temperature, Fig. 4. In this case, a thick layer of B-rich liquid could be stable on a surface (or container interface) of the A-rich liquid. There would be a diffuse-interface between the two liquids, and then near to the surface of the B-rich liquid would be a gradual enrichment to the final composition, perhaps being nearly pure $\mathrm{B}$, if it gives the lower surface energy. Then, the integrals giving the excess energy can be split to give expressions that correspond to the sum of $\gamma_{\alpha \beta}+\gamma_{\beta c}$ as follows

$$
\gamma=\frac{4}{3}\left|c_{\beta}-c_{\alpha}\right| \sqrt{\kappa \Delta f_{\max }}+\Phi\left(c_{\beta}\right)+\int_{c_{\beta}}^{c_{s}}(\phi+2 \sqrt{\kappa \Delta f}) d c
$$

The first term is the energy for the liquid/liquid interface (where the details depend upon the specific double-well potential used to describe the excess volume free energy) and $\Delta f_{\max }$ is the maximum value of the energy barrier between the stable phases. It can be seen that upon heating, the interfacial energy decreases both as the difference in composition between phases diminishes and as the free energy penalty for the material with intermediate composition decreases. It is this more rapid decrease of the interfacial energy upon heating that leads to a wetting transition, i.e., where $\gamma_{\alpha c} \rightarrow \gamma_{\alpha \beta}+\gamma_{\beta c}$. 
Qualitatively, it can be imagined that these ideas could be simply extended to two-component solid-liquid systems. Similar phenomenological treatments (sometimes termed as Landau-Ginzberg) can usefully describe aspects of premelting of solid surfaces $[9,10]$. In this instance, a wetting transition of the B-containing liquid on the A-rich solid could be expected, which may or may not occur above the eutectic temperature. If below, then it might be expected that the disordered layer could only have a finite thickness, but that it would gradually thicken giving perfect wetting at the eutectic point. However, for such a system, a single order-parameter is insufficient and several field variables including composition, structural disorder, and even orientation could be envisioned. One such generalization has been proposed to treat the solid/liquid interfacial behavior during solidification $[80,82]$. However, the present paper is more concerned with grain boundaries than surface, so we next consider analogous extensions starting first with that for a single-component grain boundary.

\section{A Phase-Field Model of Grain Boundaries}

Grain boundaries in single component systems have been successfully described with a simple phase-field model proposed by Kobayashi, Warren and Carter (KWC) [81]. An overview of that model will be given as an introduction to our recent and ongoing work on understanding transitions in grain boundaries.

Two phase-fields are used to describe the state of the system at every position

$\vec{x}$. The first field, $\vec{\theta}(\vec{x})$, is a local measure of orientation described by three rotation angles. The second field, $\eta(\vec{x})$, is a local measure of crystallinity. In two dimensions, these fields can be thought of as the coarse-grained polar 
coordinates defined by

$$
(\eta \cos \theta, \eta \sin \theta)=\frac{1}{N} \sum_{i}^{N}\left(\cos \theta_{i}, \sin \theta_{i}\right)
$$

where the sum is over all bonds $i$ in a small region that form a symmetryreduced rotation $\theta_{i}$ with a fixed coordinate system. From this definition, the bounds on each field are $0 \leq \eta \leq 1$ and $0 \leq \theta_{i} \leq \theta_{S}$ where $\theta_{S}$ is determined by symmetry. Crystalline solid corresponds to $\eta=1$ and liquid to $\eta=0$. Intermediate values of $\eta$ correspond to material states intermediate between liquid and crystalline, solid structures.

The total free energy of the system is given by an integral of the heterogeneous energy density

$$
F[\eta, \theta]=\int\left[f(\eta)+\frac{\nu^{2}}{2}(\nabla \eta)^{2}+g(\eta) s|\nabla \theta|+h(\eta) \frac{\epsilon^{2}}{2}(\nabla \theta)^{2}\right] d V
$$

where $\nu^{2}, \epsilon^{2}$ and $s$ are constants and $g(\eta)$ is a monotonically increasing function with $g(\eta=0)=0$ and $h(\eta)$ is a constant or increasing function of $\eta$. The heterogeneous free energy density has a contribution from the local value of the fields, $f(\eta)$, and the local value of the gradients in the fields. The leading order term for $\nabla \theta$ is first order which is necessary to stabilize finite (vs infinite) grain boundary widths. The second order term is necessary for the dynamic solutions; and typically it may be expected that $h(\eta)=1$ or $h(\eta=0)=0$.

For specific functions $f(\eta), g(\eta)$, and $h(\eta)$, the equilibrium grain boundary profiles in one dimension can be found, either analytically or numerically. In each case with $h(\eta)=0$, the equilibrium profile for orientation is a step 
function and the crystallinity profile has a minimum, $\eta_{0}$ at $x=0$, Fig. 5 . Further, this model admits grain rotation and for appropriate choices of $g(\eta)$, the Read-Shockley form of the low-angle to high-angle grain boundary energy function is reproduced.

The behavior of boundaries near the melting point was explored by Lobkovsky and Warren with numerics based on their sharp interface limit analysis of the KWC model $[83,84]$. They demonstrate grain boundary motion by curvature in proportion to the energy per unit length boundary. Grain boundary thickness transitions were explored near the melting point, $T_{m}$, for different choices of $h(\eta)$. With $h(\eta)=\eta^{2}$ low angle boundaries, $\Delta \theta \leq \Delta \theta_{c}$ remain narrow right to $T_{m}$ whereas high angle boundaries, $\Delta \theta>\Delta \theta_{c}$, develop a disordered layer with thickness that diverges logarithmically as $T \rightarrow T_{m}$. For $h(\eta)=1$, there are two transitions in $\Delta \theta$ at $\Delta \theta_{l}$ and $\Delta \theta_{u}$. For low angle boundaries, the boundaries remain narrow until melting at $T=T_{m}$; for high angle boundaries, there is a gradual increase in the width which diverges logarithmically as $T \rightarrow T_{m}$; for intermediate $\Delta \theta_{l} \leq \Delta \theta \leq \Delta \theta_{u}$, there is a first order transition between the narrow boundaries and wide near-liquid films at $T_{l} \leq T_{m}$.

The above analysis of wetting in a system with a heterogeneous energy density was based on the sharp interface limit. Our goal is to use the diffuse-interface formulation directly to investigate grain boundary structures and transitions.

\section{Grain Boundaries in Model Systems}

Tang has extended the analysis of transitions at grain boundaries in singlecomponent systems using a method akin to Cahn's described above [75]. The 
KWC phase-field model for grain boundaries in single-component systems provides the mathematical description [81]. Using an analytic solution for $\theta$ and the equilibrium equations and boundary conditions, the energy integral is rearranged and a graphical construction is possible.

The results indicate that grain boundaries can undergo first order transitions in thickness and order and may have a critical point. Continuous pre-melting is possible for large-angle grain boundaries where the thickness diverges logarithmically as $T-T_{m} \rightarrow 0$. It is apparent from this analysis that the misorientation, $\Delta \theta$, plays an analogous role to the undersaturation of the bulk phase, $c_{\alpha}-c_{0}$, in the binary liquid. However, we note that in the critical point wetting model, the benefit of changing the terminal composition to reduce the value of $\Phi\left(c_{s}\right)$, is achieved at the expense of including a graded transition region between $B$-rich and $B$-poor regions plus the penalty of having some volume of material with a non-equilibrium composition. For the KWC model, the analogous trade-off involves a similar penalty of having a graded region involving non-equilibrium disordered material, but this can effectively disorder the grain boundary core which completely eliminates the penalties associated with the $\nabla \theta$ terms, which may be interpreted as strain energy for a low temperature boundary. As a result of this stronger driving force, pre-melting initiates at temperatures well below that for stable liquid, the bulk melting point.

In further work, Tang has examined grain boundaries in a binary solid system using the two-component extension of the KWC model [76]. The binary model is based on a free energy expression with a homogeneous energy density, $f(\eta, c)$, that has contributions from crystallinity, $\eta$, and the concentration of $\mathrm{B}$ atoms, $c$, and with an additional gradient penalty for non-uniform composition, $\frac{\kappa^{2}}{2}(\nabla c)^{2}$, with constant coefficient $\kappa^{2}$ as well as having the gradient 
penalty terms in the previous.

Following a similar tack to that used for the single component grain boundary analysis, the step function solution for the orientation field, the boundary conditions, and the equilibrium equations are used to manipulate the energy integral into a convenient form. Due to the increased number of degrees of freedom, the graphical construction is more complicated. Tang finds both discontinuous and continuous changes in order and composition, $\eta_{0}$ and $c_{0}$, at $x=0$. As before, the misorientation plays a crucial role in determining the range of structures available for a boundary. Again disorder of the grain boundary core is favored above some temperature for high angle boundaries in order to offset the strain energy for an ordered boundary, and moreover, this trade-off can be promoted by the adsorption of solute that tends to stabilize disordered or liquid phases.

\section{Grain Boundary Films in Silicon Nitride}

Intergranular glassy films at grain boundaries in silicon nitride ceramics have been observed by many groups and are thought to be equilibrium structures $[85,86,55,87,88,56,52,89]$. Measurements of the films indicate nonuniform structure and thicknesses of 1-2nm dictated by the bulk chemistry of the silicon nitride ceramic. In addition, the average composition of the films differs from that at the intergranular pockets where grain boundaries meet. Recent high-angle annular dark-field scanning transmission electron microscopy results indicate that there is site-specific adsorption of rare-earth elements $(\mathrm{Y}, \mathrm{La}, \mathrm{Lu}, \mathrm{Sc}, \mathrm{Yb}, \mathrm{Sm})$ to grain/film and grain/pocket transition regions $[90-$ 93]. In addition, recent electron diffraction studies affirm that the films are 
inhomogeneous in terms of having structure and composition gradients across them [94].

Several models based on Clarke's approach of a force balance across a planar film between two rigid grains have been employed to understand the equilibrium nature of the films [95-97]. While these models examine the coupling of two of induced structure, chemistry, and electrostatics, and how it stabilizes a finite amorphous layer, none looks at the fully coupled problem with a complete geometric description of a grain boundary, and so none describes the behavior as a function of temperature or for situations with compositions below equilibrium liquid coexistence, i.e., for sub-eutectic temperatures or for sub-solidus compositions.

In ceramic systems, the study of equilibrium structures around necessary defects such as grain boundaries is complicated due to the charge carried by defects in these ionic materials. Locally charged regions have been shown to be stable in systems with coupled electrostatic and mechanical or chemical energetics $[98,99]$. Bishop proposed a phase-field model for grain boundaries in ceramics in her thesis [77]. That model has recently been revised to provide bulk equilibrium defect populations, something typically neglected in bulk thermodynamic treatments such as that described in Section 1 [78].

The current model is based on a multi-component KWC model so that it can provide a 3-D theoretical framework for examining grain boundary behavior in multicomponent systems with charged defects. The independent fields are $\eta(\vec{x})$ and $\vec{\theta}(\vec{x})$ as defined for KWC, $X_{i}(\vec{x})(i=1,2, \ldots, N)$ the site fraction of neutral species, $X_{j}^{d}(\vec{x})(j=1,2, \ldots, M)$ the site fraction of defects which carry a formal charge of $Z_{j}^{d} e$ each where $e$ is the magnitude of the charge on an 
electron, and $\phi(\vec{x})$ the electrostatic potential. There is a uniform site density, $\frac{1}{V_{m}}$. The total free energy is given by

$$
\begin{aligned}
F\left[X_{i}, X_{j}^{d}, \phi, \eta, \vec{\theta} ; T, V\right]= & \int_{V}\left[f\left(X_{i}, X_{j}^{d}, \eta ; T\right)\right. \\
& +\rho \phi-\frac{1}{2} \epsilon(\nabla \phi)^{2} \\
& +\frac{1}{2} \sum_{i=1}^{N} \kappa_{i}^{2}\left(\nabla X_{i}\right)^{2}+\frac{1}{2} \sum_{j=1}^{M} \kappa_{j}^{2}\left(\nabla X_{j}^{d}\right)^{2} \\
& \left.+\frac{1}{2} s g(\eta)|\nabla \vec{\theta}|+\frac{1}{2} \beta^{2} h(\eta)(\nabla \vec{\theta})^{2}+\frac{1}{2} \nu^{2}(\nabla \eta)^{2}\right] d \vec{x}
\end{aligned}
$$

where $\rho=\frac{\mathcal{F}}{V_{m}} \sum_{j}^{M} Z_{j}^{d} X_{j}^{d}$ is the charge density with $\mathcal{F}$ Faraday's constant. The constant, isotropic dielectric constant is $\epsilon$. The gradient energy parameters are $s, \nu^{2}, \beta^{2}, \kappa_{i}^{2}$, and $\kappa_{j}^{2}$. The functions $g(\eta)$ and $h(\eta)$ weight the gradient contributions from non-uniform $\vec{\theta}(\vec{x})$. A full justification of this model is available elsewhere [77].

Preliminary results for calculations with this model for planar grain boundaries in a point defect-free pseudo-binary $\mathrm{Si}_{3} \mathrm{~N}_{4}-\mathrm{SiO}_{2}$ approximation and taking $h(\eta)=1$ indicate that intergranular films can be stabilized at high-angle grain boundaries below the temperature at which bulk-liquid is stable. Our calculations find sharp, low-adsorption boundaries and wide, disordered, highadsorption boundaries at different values of misorientation, temperature, and chemical potential, Fig. 6 . We also find perfect wetting at temperatures above the eutectic and for chemical potentials corresponding to bulk coexistence of $\mathrm{Si}_{3} \mathrm{~N}_{4}$-rich solid and $\mathrm{SiO}_{2}$-rich liquid. The transitions from thin to thick films have not been resolved sufficiently in order to distinguish if they are continuous or discontinuous in nature. This work is anticipated in the future.

In addition to the several fields and associated gradient terms and the simplifications described here, i.e. the minimal defect description and the constant 
site density and isotropic dielectric constant, it is anticipated that dispersion forces will play a role in the equilibrium thickness and structure of IGFs [100]. For symmetric systems, the effect of the dispersion force will be attractive between the grain surfaces, resulting in a thinner equilibrium film thickness, although it is debatable whether this would be enough to keep finite film thicknesses at all conditions. While it is possible to write the London dispersion force contribution to the energetics for a simple 121 slab configuration, $E_{\text {disp }}=\frac{A_{121}}{12 \pi h^{2}}$, using a Hamaker constant $A_{121}$ which depends on the local optical properties and $h$ the thickness of the film, it is not straightforward to include this in the variational approach to solving for equilibrium structures in phase-field models. This problem can be overcome by using a direct minimization method such as simulated annealing [101]. The effects of having a strong adsorption near the crystalline/disorder transition of a disordered layer can be better approximated using the analogous 12321 approximation in which the graded region is approximated as a separate layer regarding the influence on dispersion forces [100].

\section{Tuning Phase-Field Models to Materials Systems}

The preliminary results for the phase-field model described in Section 5 show behavior that can be modified by the choice of the gradient energy coefficients and the functional form of $g(\eta)$ and $h(\eta)$. There are no standard methods for tuning model parameters to reflect experimental data in real systems. Some ideas on this topic are discussed here.

It is necessary to compute an energy density corresponding to states that exist only in the vicinity of interfaces but not in the bulk. There is some freedom 
as to how to interpolate the free energy density between the known values for liquids and solids. We use a scheme based on that proposed by Wheeler for a model system [102] and subsequently used to couple phase-field models to thermodynamic databases $[103,104]$.

In the present work, we incorporate bulk thermodynamic data into the chemical contributions to the homogeneous free energy density, $f\left(X_{i}, X_{j}^{d}, \eta ; T\right)[78]$. For simplicity, the concept is illustrated for a binary system where $X_{B}$ is the concentration of B atoms. The homogeneous free energy density for a binary system is a function of $X_{B}$ and the liquid-solid phase field, $\eta$

$$
\begin{aligned}
f\left(X_{B}, \eta\right)= & X_{B} f_{B, 0}(\eta ; T)+\left(1-X_{B}\right) f_{A, 0}(\eta ; T) \\
& \frac{R T}{V_{m}}\left[X_{B} \ln X_{B}+\left(1-X_{B}\right) \ln \left(1-X_{B}\right)\right]+\Omega(\eta ; T) X_{B}\left(1-X_{B}\right)
\end{aligned}
$$

with free energies of formation of the pure components, $f_{A, 0}$ and $f_{B, 0}$, and a coefficient for the free energy of mixing, $\Omega$, that depend on $\eta$ and temperature. A double-well form is used for the $\eta$-dependence of the free energies of formation that interpolates between the free energy of formation of the liquid and solid phases at $T$ with a single parameter that controls the height of the energy barrier.

The precise details of the profiles of the fields across a grain boundary are effected by the choice of model parameters: gradient energy coefficients, coefficients on enthalpy of mixing, and energy barriers in free energies of formation. Koch proposed a scheme to adjust these free parameters to available information using a simulated annealing technique with a least squares figure of merit depending on a comparison of equilibrium field profiles for a given set of model parameters [94]. However, there is some tension to be resolved be- 
tween optimizing the parameters to best describe a given interface at a given temperature and to best describe the broad behavior over the whole range of composition and temperature space.

Experimental field profiles are typically continuum measures, e.g. composition from EELS scans across boundaries. These data can be compared directly to the continuum fields from a phase-field model. There is additional data available in atomistic or first principles calculations, for example on IGFs in silicon nitride [105-108]. In order to use this data to tune phase-field models it must be continuized.

The KWC definitions of $\eta$ and $\vec{\theta}$, Eq. 5 , are not easily generalized to multicomponent systems where the definition of $\overrightarrow{\theta_{i}}$ is unclear. In continuizing fields for composition, the only necessary step is to average local values in space. In continuizing fields like $\eta$, it is first necessary to define $\eta$ for each atom and then average in space.

Bishop proposed methods to calculate local measures of structure and continuize in single-component, single crystalline phase materials [109,77]. These methods depend on coarse-graining local measures of atomic structure centered on atoms. One technique measures the local structure as a comparison of geometric properties of the Voronoi tessellations (VTs) of the atoms. The second compares the bond angle distribution functions (BADFs) of atoms with mathematically defined neighbors. In each case, the local measures are compared to the same measure for an atom in a perfect crystal in order to generate a measure of $\eta$. The data associated with each atom is assigned to the Voronoi tessellation for each atom (this fills space) and then averaged within a sphere centered at a position $\vec{x}$ to generate $\eta(\vec{x})$. Examples of linear profiles for 
a simulated grain boundary in $\mathrm{Si}$ in Fig. 7 illustrate that the general features of the two techniques are similar but that the VTs are more sensitive to small shifts in atomic positions. The different scales are arbitrary.

The BADFs seem to give a better method and one that could be extended more easily to multi-component systems using partial neighbor lists. This technique depends on there being a single equilibrium crystal structure with which to compare the local BADF. However, in phase-field models of grain boundaries with multiple possible crystal structures an additional phase-field must be introduced to distinguish between the crystal structures in the energetics. Therefore, the BADF's could be used to measure the closest BADF match to the available crystal structures and the BADF distance from it. These ideas are the subject of future work.

\section{$7 \quad$ Summary and Outlook}

Advances in understanding possible grain boundary composition and structure profiles and structure transitions in single- and binary-component materials have been made with energetics modeled with the KWC phase-field model and generalizations of it. That analysis utilizes diffuse-interface concepts inspired by Cahn's critical point wetting work. It has allowed the exploration of stable and metastable grain boundary compositions and structures. A general phase-field model has also been developed that allows the couplings between chemistry, structure, and electrostatics to be explored for application to ionic and covalent components.

The models reviewed in this paper are extensions of classical diffuse-interface 
theory to fixed stoichiometry and multicomponent systems with grain boundaries. They rely on two additional fields that contribute to a coarse-grained model for energy density beyond that depending only on the local crystalline orientation and a measure of crystalline disorder. The continuum model and numerical calculations reviewed in this paper are developed from model or experimental thermodynamic data for specific alloy systems. These models include physical characteristics of grain boundaries and therefore include predictions of physical phenomena that depend on these characteristics, such as grain boundary disorder and segregation as a function of misorientation or temperature. The crystalline orientation is routinely measured by diffraction techniques. Crystalline disorder is a necessary component in the diffuseinterface theory, but its experimental verification is troublesome and is a matter of current investigation. As a consequence, the model has some general trends of behavior, but can be tuned to exhibit system-specific attributes.

For the KWC model, there is a general trend toward pre-melting of a highangle boundary with disorder increasing well below the eutectic or melting temperature. As presently formulated, liquids tend to fully wet the boundary as the temperature approaches the melting or eutectic point. The amount of core disorder, the core composition, and the characteristic width of the disordered grain boundary are equilibrium thermodynamic parameters - there are no extra degrees of freedom associated with the boundary. The disorder and composition of the core and the characteristic width are functions of the bulk composition, temperature, and boundary misorientation. As such the model produces general trends that match certain highly simplified lattice calculations such as those by Kikuchi and Cahn [26,27], but the robust trends can be tuned to be system specific. The grain boundary wetting can be delayed 
until higher temperatures for grain boundaries by inclusion of the London dispersion forces, which are composition dependent, as described.

Because the grain boundary has its own characteristic equilibrium state, it has the attributes of a two dimensional phase. From this perspective, the diffuse-interface theory predicts grain boundary complexion transitions where, at specific compositions and temperatures, the boundary has two equilibrium states. Therefore, for some boundaries at a range of compositions, there is a first order transition in the boundary state with temperature. There are a range of predicted behaviors for core disorder and boundary width as the grain boundary temperature increases towards the bulk melting temperature, and these have been rationalized by observation and simple models.

These models provide an interfacial analog to the theory and observation of critical wetting on surfaces. We believe that this interfacial analog is related to experimental observations of stable grain boundary films in many ceramic systems, as have been extensively studied with $\mathrm{Si}_{3} \mathrm{~N}_{4}$ and model binary oxides. The continuum models predict that, especially in systems with significant short-range order in their liquid state, grain boundaries will disorder below the melting temperature. These disordered boundaries will have structures that resemble liquids and spread the penalty for misorientation change across the boundary over a considerable width. The extensively observed nanometer wide disordered boundaries seen in these Si-based and oxide ceramics are consistent with the types of predicted behavior for KWC-based models. However, the experimental trends are that often the disordered boundaries initiate well below bulk melting, but complete wetting is delayed until well above bulk melting. Thus there must be two transition temperatures in contrast to original critical point wetting theory which gives only one. 
As mentioned, this delay in wetting can formally derive from the London dispersion forces. However, space charge effects in certain instances can also exert a stabilizing influence of being attractive and resisting the other disjoining forces that derive from the gradient penalties. From colloid theory it is generally expected that the space charge effects will tend to be repulsive $[110,96]$. However, it has recently been shown that there can be an attractive regime especially in materials in which the dielectric coefficients are high [111]. If important, it would imply fundamentally different behavior could obtain in ionic or covalent materials versus metals, which is not yet established. Another extension to be explored in this regard is inclusion of coherence types of effects in which the transition region of the disordered material inherits significant order from the neighboring lattice. Finally, the models, as presented, have a similar deficiency as the original critical point wetting model; at lower temperature there is only a weak tendency for surface adsorption, and it tends to increase with heating in contrast to that in the more common behavior of Langmuir-McLean-like models. Modifications to better include such effects are being incorporated into the phase-field models for evaluation.

The models are interface analogues of the better studied surface roughening and pre-melting behavior. The TEM evidence shows pure metallic grain boundaries tend to preferentially melt, but only above $0.999 T_{m}$ in terms of forming observable layers thicker than 1-2 nm. This may be consistent with the Kikucki and Cahn lattice gas model $[26,27]$ and the recent analysis of the KWC model described here in which the thickness increases with $-\ln \left(T_{m}-T\right)$, and so only becomes appreciably thick very close to the bulk melting point. By comparison, for free surfaces the roughening transition can initiate somewhere from 0.5 to $0.7 T_{m}$, depending upon the orientation, and at $1^{\circ} \mathrm{C}$ undercool- 
ing the pre-melted/disordered layer can be 5 monolayers thick, again about a nanometer. Such behavior has also been shown to be consistent with similar phenomenological diffuse-interface concepts $[9,10]$.

The more interesting issue relates to the onset of disorder, comparable to the roughening transition that initiates at lower temperatures. For pure metals, in grain boundaries with sufficiently special misorientations that secondary grain boundary dislocations are localized, this localization does not change up to $0.96 T_{m}$ [29], suggesting the roughening transition is delayed versus that for surfaces. However, is has also been observed that defaceting transitions of pure grain boundaries can initiate at 0.5 to $0.9 T_{m}$, indicating that some degree of disorder has occurred [31,32]. However, the transitions in the models may be better related to roughening transitions that initiate on surfaces at 0.5 to $0.67 T_{m}$. In this regard, for a high angle boundary, it may be that these transitions should be considered by analogy to glass transitions in which the change is from a rather static amorphous structure in a low energy configuration to a dynamic structure sampling many configurations and having a much higher point defect content. Exploring and elucidating such concepts will challenge the experimental (e.g., TEM) as well as theory communities.

That grain boundary disordering transitions have not been extensively catalogued in metals may be attributed to the nature of the transition or to differences between metals and ceramic systems. If first-order transitions exist at grain boundaries in metals, they may entail a very small discontinuous change in core structure and that structure may have high crystallinity which could make it difficult to resolve in the TEM. Also, the analysis shows that in materials with a small energy barrier between crystalline and liquid states, the transition can be second-order and again, difficult to characterise. It is 
expected that metallic systems near $T_{m}$ have this property. In contrast, the analysis suggests that ceramics may demonstrate disordered grain boundaries at lower temperatures than in metals due to the small energy barrier between crystalline solids and disordered glass-forming compositions such as silicates. In addition, as these glass formers can have relatively complicated structures and so larger structureal units, the width of the disordered regions may make them easier to characterise in the TEM.

More complex behavior may derive for binary systems as indicated by the extensive literature on enhanced diffusion, grain growth or sintering that can occur well below the eutectic temperature. Analogous behavior has been seen for a nanometer thick surface film that forms on a specific surface in the $\mathrm{ZnO}-\mathrm{Bi}_{2} \mathrm{O}_{3}$ system that is analogous to the amorphous grain boundaries as seen; these films gradually thicken with temperature within a wide range of temperature, which is bounded by two transitions and also spans the eutectic [112-114]. Below the multilayer or drying transition, these multilayer films are unstable, and they disappear with suitable annealing.

For specific interfaces and under limited conditions, atomistic calculations produce better interfacial energy and structural data than continuum models. Continuum models are less precise, but they predict comprehensive behavior for generic boundaries over wide ranges of thermodynamic variables. The parameters that are employed in the coarse-grained continuum or diffuseinterface models are empirical. Methods have been developed to obtain these parameters from atomistic data sets. The data reduction methods are promising because they abstract information from more precise calculations, but empirical models are still required to extrapolate coarse-grained parameters to environmental conditions where atomistic calculations cannot be performed. 
Furthermore, the coarse graining methods necessarily include arbitrary choices of algorithm which are inherent in any fitting technique. Careful consideration and communication of the coarse-graining technique will become a necessary part of experimental characterization. The coarse-graining techniques also provide methods to compare atomistic data to experiments that average behavior over a finite region.

Numerical calculations, based on the thermodynamics that generate the Si-O$\mathrm{N}$ phase diagram, show behavior that is consistent with observations in that system. Furthermore, they predict other properties, such as defect concentration and segregation that could be experimentally verified.

The continuum models provide a means to understand observed behavior in a variety of systems. Some methods have been developed to compare them to experiments and atomistic calculations, although much remains to be investigated. Predictive continuum models will produce interfacial phase diagrams that will be useful for materials design, and may provide guidance on particular systems onto which more precise computational resources may be applied. If sufficiently accurate, such models have the advantage that they can not only describe equilibrium interfacial complexions and their transitions over the broad range of temperature, pressure, and composition space, but that they can also be readily used to provide quantitative models for kinetic and dynamic behaviors in chemically heterogeneous systems during processes such as phase transformations, grain coarsening or fluid spreading. 


\section{Acknowledgement}

CMB, MT, and WCC acknowledge support from NSF Grant DMR-0010062. RMC was supported by the Office of Basic Energy Science, Division of Materials Sciences and Engineering of Dept. of Energy under Contract No. DE-AC0376F00098. The work described here was conducted in cooperation with the whole NANOAM collaboration whose European partners were funded from EU Commission Contract G5RD-CT-2001-00586. The discussions and hospitality of Dr. Dominique Chatain and of Profs. Adrian Sutton and David Cockayne are gratefully acknowledged by WCC and CMB.

\section{References}

[1] W. K. Burton, N. Cabrera, F. C. Frank, The growth of crystals and the equilibrium structure of their surfaces, Philosophical Transactions of the Royal Society of London. Series A, Mathematical and Physical Sciences 243 (1951) 299-358.

[2] H. J. Leamy, G. H. Gilmer, K. A. Jackson, Statistical thermodynamics of clean surfaces, in: J. M. Blakely (Ed.), Surface Physics of Materials, Academic Press, New York, 1975, pp. 121-188.

[3] R. J. Brehm, G. Ertl, V. Penka, R. Schwanker, The Structure of Surfaces, Springer, Berlin, 1985.

[4] R. Vanselow, R. F. Howe (Eds.), Chemistry and Physics of Solid Surfaces, Springer-Verlag, Berlin, 1988.

[5] H. Taub, G. Torzo, H. J. Lauter, S. C. Fain-Jr. (Eds.), Phase Transitions in Surface Films 2. NATO ASI Series, Plenum Press, New York, 1991. 
[6] J. Lapujoulade, B. Salanon, F. Fabre, B. Loisel, The roughening transition of vicinal surfaces, in: M. G. Lagally (Ed.), Kinetics of Ordering and Growth at Surfaces, Plenum Press, New York, 1990, pp. 355-368.

[7] J. G. Dash, Surface melting, Cont. Phys. 30 (1989) 89-100.

[8] J. G. Dash, H. Fu, J. S. Wettlaufer, The premelting of ice and its environmental consequences, Reports on Progress in Physics 58 (1995) 115-167.

[9] J. F. van-der Veen, B. Pluis, A. W. D. van-der Gon, Ion scattering studies of surface melting, in: M. G. Lagally (Ed.), Kinetics of Ordering and Growth at Surfaces, Plenum Press, New York, 1990, pp. 343-354.

[10] J. F. van-der Veen, Surface induced melting in solids, in: H. Taub (Ed.), Phase Transitions in Surface Films 2, Plenum Press, New York, 1991, pp. 289-305.

[11] R. H. Fowler, Statistical Mechanics, Cambridge University Press, Cambridge, UK, 1936 .

[12] J. M. Blakely, H. V. Thapliyal, Structure and phase transitions of segregated surface layers, in: Interfacial Segregation, ASM Seminar, American Society for Metals, Metal Park, OH, 1978, pp. 137-174.

[13] R. Feidenhaus, F. Grey, M. Nielsen, R. L. Johnson, Structure and ordering of metal overlayers on $\operatorname{Si}(111)$ and $\mathrm{Ge}(111)$ surfaces, in: M. G. Lagally (Ed.), Kinetics of Ordering and Growth at Surfaces, Plenum Press, New York, 1990, pp. 189-207.

[14] S. G. F. Mochrie, D. Gibbs, D. M. Zehner, Phase behavior of monolayers, in: D. Wolf, S. Yip (Eds.), Materials Interfaces, Plenum Press, London, UK, 1992, pp. $336-353$.

[15] W. A. Steele, Monolayers of linear molecules adsorbed on the graphite basal plane: Structures and intermolecular interactions, Langmuir 12 (1996) 145153. 
[16] R. Pandit, M. Schick, M. Wortis, Systematics of multilayer adsorption phenomena on attractive substrates, Physical Review B 26 (1982) 5112-5140.

[17] M. Schick, Introduction to wetting phenomena, in: J. Charvolin, et al. (Eds.), Liquids at Interfaces, Elsevier, Amsterdam, the Netherlands, 1990, pp. 415497.

[18] M. Wortis, Equilibrium crystal shapes and interfacial phase transitions, in: R. Vanselow, R. F. Howe (Eds.), Chemistry and Physics of Solid Surfaces VII, Springer-Verlag, Berlin, 1988, pp. 376-405.

[19] P. Wynblat, Coupled chemical and faceting surface phase transitions, in: P. M. Duxbury, T. J. Pence (Eds.), Dynamics of Crystal Surfaces and Interfaces, Plenum Press, New York, 1997, pp. 231-238.

[20] J. W. Cahn, Critical point wetting, Journal of Chemical Physics 66 (1977) $3667-3672$.

[21] M. R. Moldover, J. W. Cahn, An interface phase transition: complete to partial wetting, Science 207 (1980) 1073-1075.

[22] S. Dietrich, Fluid interfaces:wetting, critical adsorption, van der Waals tails, and the concept of the effective interface potential, in: H. Taub, et al. (Eds.), Phase Transitions in Surface Films 2, Plenum Press, New York, 1991, pp. $391-423$.

[23] E. W. Hart, Two-dimensional phase transformation in grain boundaries, Scripta Metall. 2 (1968) 179-182.

[24] J. W. Cahn, Transitions and phase equilibria among grain boundary structures, J. Physique 4 (1982) 199-213.

[25] E. I. Rabkin, L. S. Shvindlerman, B. B. Straumal, Grain boundaries: Phase transitions and critical phenomena, Int. J. of Mod. Phys. B5 (1991) 2989-3028. 
[26] R. Kikuchi, J. W. Cahn, Grain-boundary melting transition in a twodimensional lattice-gas model, Physical Review B 21 (1980) 1893-1897.

[27] R. Kikuchi, J. W. Cahn, Grain-boundaries with impurities in a twodimensional lattice-gas model, Physical Review B 36 (1987) 418-428.

[28] C. Rottman, Thermal fluctuations in interfaces: From fluid-fluid interfaces to small-angle grain boundaries, Mater. Sci. Eng. 81 (1986) 553-562.

[29] T. E. Hsieh, R. W. Balluffi, Experimental study of grain boundary melting in aluminum, Acta Metall. 37 (1989) 1637-1644.

[30] A. P. Sutton, R. W. Balluffi, Interfaces in Crystalline Materials, Clarenden Press, Oxford, UK, 1995, chapters 6-7.

[31] T. G. Ference, R. W. Balluffi, Observations of a reversible grain boundary faceting transition induced by changes of composition, Scripta Metall. 22 (1988) 1929-1934.

[32] T. E. Hsieh, R. W. Balluffi, Observations of roughening/defaceting phase transitions in grain boundaries, Acta Metall. 37 (1989) 2133-2139.

[33] R. W. Balluffi, Grain boundary structure and segregation, in: Interfacial Segregation, ASM Seminar, American Society for Metals, Metal Park, OH, 1978, pp. 193-236.

[34] L.-S. Chang, E. I. Rabkin, B. Straumal, P. Lejcek, S. Hofmann, W. Gust, Temperature dependence of the grain boundary segregation of $\mathrm{Bi}$ in $\mathrm{Cu}$ polycrystals, Scripta Mater. 37 (1997) 729-735.

[35] L.-S. Chang, E. I. Rabkin, B. B. Straumal, B. Baretzky, W. Gust, Thermodynamic aspects of the grain boundary segregation in $\mathrm{Cu}(\mathrm{Bi})$ alloys, Acta Mater. 47 (1999) 4041-4046. 
[36] L.-S. Chang, E. Rabkin, B. B. Straumal, S. Hofmann, B. Baretzky, W. Gust, Grain boundary segregation in the cu-b system, Defect Diff. Forum 156 (1998) $135-146$.

[37] H. Gleiter, Observations suggesting a transformation in the structure of highangle grain boundaries in lead, Z. Metallkd 61 (1970) 282-297.

[38] E. L. Maksimova, E. I. Rabkin, L. S. Shvindlerman, B. B. Straumal, Phase transitions at grain boundaries in the presence of impurities, Acta Metall. 37 (1989) 1995-1998.

[39] E. I. Rabkin, L. S. Shvindlerman, B. B. Straumal, W. Gust, Grain boundaries: Phase transitions and critical phenomena, Mater. Sci. Forum 126-28 (1993) 305-313.

[40] J. Schölhammer, B. Baretzky, W. Gust, E. Mittemeijer, B. Straumal, Grain boundary grooving as an indicator of grain boundary phase transition, Interface Sci. 9 (2001) 41-51.

[41] M. B. Kasen, Some observations on boundary segregation during grain growth annealing of ultrapurity aluminum, Acta Metall. 20 (1972) 105-13.

[42] C. J. Simpson, W. C. Winegard, K. T. Aust, Grain boundary migration and boundary-solute interactions, in: G. A. Chadwick, D. A. Smith (Eds.), Grain Boundary Structure and Properties, Academic Press, New York, 1976, pp. 201-234.

[43] U. Erb, H. Gleiter, The effect of temperature on the energy and structure of grain boundaries, Scripta Metall. 13 (1979) 61-64.

[44] D. A. Molodov, U. Czubayko, G. Gottstein, L. S. Shvindlerman, B. Straumal, W. Gust, Acceleration of grain boundary motion in Al by small additions of Ga, Philos. Mag. Lett. 72 (1995) 361-368. 
[45] D. Weygand, Y. Brechet, E. Rabkin, B. Straumal, W. Gust, Solute drag and wetting of a grain boundary, Philos. Mag. Lett. 76 (1997) 133-138.

[46] S. I. Bae, S. Baik, Determination of critical concentrations of silica and/or calcia for abnormal grain growth in alumina, J. Am. Ceram. Soc. 76 (1993) $1065-1067$.

[47] I. MacLaren, R. M. Cannon, M. A. Gülgün, R. Voytovych, N. Popescu-Pogrion, C. Scheu, U. Täffner, M. Rühle, Abnormal grain growth in alumina: Synergistic effects of yttria and silica, J. Am. Ceram. Soc. 86 (2003) 650-59.

[48] R. L. Coble, R. M. Cannon, Current paradigms in powder processing, in: E. A. H. P. III (Ed.), Processing of Crystalline Ceramics, Plenum Press, New York, 1978, pp. 151-168,170.

[49] J. Luo, H. Wang, Y.-M. Chiang, Origin of solid-state activated sintering in $\mathrm{Bi}_{2} \mathrm{O}_{3}$-doped $\mathrm{ZnO}$, Journal of the American Ceramic Society 82 (1999) 916920 .

[50] H.-J. Kleebe, Structure and chemistry of interfaces in $\mathrm{Si}_{3} \mathrm{~N}_{4}$ materials studied by transmission electron microscopy, J. Ceram. Soc. Jpn 105 (1997) 453-475.

[51] R. M. Cannon, L. Esposito, High temperature colloidal behavior: Particles in liquid silicates, Zeitschrift für Metallkunde 90 (1999) 1002-1015.

[52] R. M. Cannon, M. Rühle, M. J. Hoffmann, R. H. French, H. Gu, A. P. Tomsia, E. Saiz, Adsorption and wetting mechanisms at ceramic grain boundaries, Ceram. Trans. 118 (2000) 427-444.

[53] A. Subramaniam, C. T. Koch, M. Rühle, R. M. Cannon, Intergranular glassy films: An overview, submitted to Mater. Sci. Eng. (2005).

[54] H.-J. Kleebe, M. K. Cinibulk, R. M. Cannon, M. Rühle, Statistical analysis of the intergranular film thickness in silicon nitride ceramics, J. Am. Ceram. Soc. 76 (1993) 1969-1977. 
[55] I. Tanaka, H.-J. Kleebe, M. K. Cinibulk, J. Bruley, D. R. Clarke, M. Rühle, Calcium concentration dependence of the intergranular film thickness in silicon nitride, Journal of the American Ceramic Society 77 (1994) 911-914.

[56] C.-M. Wang, X. Pan, M. J. Hoffmann, R. M. Cannon, M. Rühle, Grain boundary films in rare-earth-based silicon nitride, Journal of the American Ceramic Society 79 (1996) 788-792.

[57] H.-J. Kleebe, $\mathrm{SiC}$ and $\mathrm{Si}_{3} \mathrm{~N}_{4}$ materials with improved fracture resistance, Journal European Ceramic Society 10 (1992) 151-159.

[58] J. J. Cao, W. J. MoberlyChan, L. C. DeJonghe, C. J. Gilbert, R. O. Ritchie, In situ toughened silicon carbide with Al-B-C additions, Journal American Ceramic Society 79 (1996) 461-469.

[59] D. Chen, X.-F. Zhang, R. O. Ritchie, Effects of grain-boundary stucture on strength, toughness, and cyclic-fatigue properties of a monolithic silicon carbide, Journal American Ceramic Society 83 (2000) 2079-2081.

[60] Y.-M. Chiang, L. A. Silverman, R. H. French, R. M. Cannon, Thin glass film between ultrafine conductor particles in thick-film resistors, J. Am. Ceram. Soc. 77 (1994) 1143-1152.

[61] D.-Y. Kim, S. M. Wiederhorn, B. J. Hockey, J. E. Blendell, C. A. Handwerker, Stability and surface energies of wetted grain boundaries in aluminum oxide, J. Am. Ceram. Soc. 77 (1994) 444-453.

[62] R. Brydson, S.-C. Chen, F. L. Riley, S. J. Milne, X. Pan, M. Rühle, Microstructure and chemistry of intergranular glassy films in liquid-phasesintered alumina, J. Am. Ceram. Soc. 81 (1998) 369-379.

[63] J.-R. Lee, Y.-M. Chiang, Bi segregation at Zno grain boundaries in equilibrium with $\mathrm{Bi}_{2} \mathrm{O}_{3}-\mathrm{ZnO}$ liquid, Sol. St. Ionics 75 (1995) 79-88. 
[64] J.-R. Lee, Y.-M. Chiang, Pressure-thermodynamic study of grain boundaries: Bi in ZnO, Acta Mater. 45 (1997) 1247-1257.

[65] Y.-M. Chiang, H. Wang, J.-R. Lee, HREM and STEM of intergranular films at zinc oxide varistor grain boundaries, J. Microscp. 191 (1998) 275-85.

[66] S. Ramamurthy, M. P. Mallamaci, C. M. Zimmerman, C. B. Carter, P. R. Duncombe, T. M. Shaw, Microstructure of polycrystalline $\mathrm{MgO}$ penetrated by a silicate liquid, JMSA ISSN 2 (1996) 113-128.

[67] J. J. Kim, M. P. Harmer, Effect of liquid volume fraction on grain growth of magnesium oxide grains in molten calcium magnesium silicate matrix, J. Am. Ceram. Soc. 84 (2001) 3027-3031.

[68] H. Gu, R. M. Cannon, M. Rühle, Composition and chemical width of ultrathin intergranular amorphous film in silicon nitride, J. Mater. Res. 13 (1998) $476-487$.

[69] H. Gu, X. Pan, R. M. Cannon, M. Rühle, Dopant distribution in grainboundary films in calcia-doped silicon nitride ceramics, Journal of the American Ceramic Society 81 (1998) 3125-3125.

[70] H. Gu, R. M. Cannon, H. J. Seifert, M. J. Hoffmann, I. Tanaka, Solubility of $\mathrm{Si}_{3} \mathrm{~N}_{4}$ in liquid $\mathrm{SiO}_{2}$, J. Am. Ceram. Soc. 85 (2002) 25-32.

[71] H. Gu, R. M. Cannon, I. Tanaka, M. Rühle, Calcia partition in phase separated intergranular glass and interfaces in doped, submitted to Mater. Sci. Eng. (2005).

[72] B. Straumal, W. Gust, Lines of grain boundary phase transitions in bulk phases diagrams, Mater. Sci. Forum 207-209 (1996) 59-68.

[73] B. Straumal, E. Rabkin, W. Lojkowski, W. Gust, L. S. Shvindlerman, Pressure influence on the grain boundary wetting transition in Fe-Si alloys, Acta Mater. 45 (1997) 1931-1940. 
[74] J. P. Gambino, W. D. Kingery, G. E. Pike, H. R. Phillip, Effect of heat treatments on the wetting behavior of bismuth rich intergranular phases in ZnO:Bi:Co varistors, J. Am. Ceram. Soc. 72 (1989) 642-645.

[75] M. Tang, W. C. Carter, R. M. Cannon, Order-disorder structural transitions at grain boundaries in the KWC phase-field model, in preparation (2005).

[76] M. Tang, W. C. Carter, Ordering transitions at grain boundaries in twocomponent systems, in preparation (2005).

[77] C. M. Bishop, Continuum models for intergranular films in silicon nitride and comparison to atomistic simulations, Ph.D. thesis, Department of Materials Science and Engineering, Massachusetts Institute of Technology (2003).

[78] C. M. Bishop, R. M. Cannon, W. C. Carter, A diffuse-interface model of interfaces:Grain boundaries in silicon nitride, Acta MaterialiaIn press.

[79] J. W. Cahn, J. E. Hilliard, Free energy of a non-uniform system. I. Interfacial free energy, The Journal of Chemical Physics 28 (1958) 258-267.

[80] J. A. Warren, W. J. Boettinger, Prediction of dendritic growth and microsegregation patterns in binary alloy using phase-field method, Acta Metallurgica and Materialia 43 (1995) 689-703.

[81] R. Kobayashi, J. A. Warren, W. C. Carter, A continuum model of grain boundaries, Physica D 140 (2000) 141-150.

[82] R. Kobayashi, J. A. Warren, W. C. Carter, A phase-field model of the impingement of solidifying particles, Physica D 261 (1998) 159-166.

[83] A. E. Lobkovsky, J. A. Warren, Sharp interface limit of a phase-field model of crystalline grains, Physical Review E 63 (2001) 051605.

[84] A. E. Lobkovsky, J. A. Warren, Phase field model of premelting of grain boundaries, Physica D 164 (2002) 202-212. 
[85] H. Gu, R. M. Cannon, M. Rühle, Composition and chemical width of ultrathin amorphous films at grain boundaries in silicon nitride, Journal of Materials Research 13 (1998) 376-387.

[86] H.-J. Kleebe, M. J. Hoffmann, M. Rühle, Influence of secondary phase chemistry on grain boundary film thickness in silicon nitride, Zeitschrift für Metallkunde 83 (1992) 610-617.

[87] I. Tanaka, J. Bruley, H. Gu, M. J. Hoffmann, H.-J. Kleebe, R. M. Cannon, D. R. Clarke, M. Rühle, Compositions and thicknesses of grain boundary films in cadoped silicon nitride ceramics, in: M. J. Hoffmann, G. Petzow (Eds.), Tailoring of Mechanical Properties of $\mathrm{Si}_{3} \mathrm{~N}_{4}$ Ceramics, Kluwer Academic Publishers, Amsterdam, 1994, pp. 275-289.

[88] H.-J. Kleebe, M. K. Cinibulk, I. Tanaka, J. Bruley, J. S. Vetrano, M. Rühle, High resolution electron microscopy studies on silicon nitride ceramics, in: M. J. Hoffmann, G. Petzow (Eds.), Tailoring of Mechanical Properties of $\mathrm{Si}_{3} \mathrm{~N}_{4}$ Ceramics, Kluwer Academic Publishers, Amsterdam, 1994, pp. 259-274.

[89] M. J. Hoffmann, H. Gu, R. M. Cannon, Influence of the interfacial properties on the microstructural development and properties of silicon nitride ceramics, in: Materials Research Society Proceedings, Vol. 586, Materials Research Society, Warrendale, PA, 2000, pp. 65-74.

[90] N. Shibata, S. J. Pennycook, T. R. Gosnell, G. S. Painter, W. A. Shelton, P. R. Becher, Observation of rare-earth segregation in silicon nitride ceramics at subnanometre dimensions, Nature 428 (2004) 730-733.

[91] A. Ziegler, J. C. Idrobo, M. K. Cinibulk, C. Kisielowski, N. D. Browning, R. O. Ritchie, Interface structure and atomic binding characteristic in silicon nitride ceramics, Science 306 (2004) 1768-1700.

[92] G. B. Winkelman, C. Dwyer, T. S. Hudson, D. Nguyen-Manh, M. Döblinger, 
R. L. Satet, M. J. Hoffmann, D. J. H. Cockayne, Arrangement of rareearth elements at prismatic grain boundaries in silicon nitride, accepted to Philosophical Magazine Letters (2004).

[93] G. B. Winkelman, C. Dwyer, unpublished research (2005).

[94] C. Koch, unpublished research.

[95] D. R. Clarke, On the equilibrium thickness of intergranular glass phases in ceramic materials, Journal of the American Ceramic Society 70 (1987) 15-22.

[96] D. R. Clarke, T. M. Shaw, A. P. Philipse, R. G. Horn, Possible electrical double-layer contribution to the equilibrium thickness of intergranular glass films in polycrystalline ceramics, Journal of the American Ceramic Society 76 (1993) 1201-1204.

[97] D. R. Clarke, The intergranular film in silicon nitride ceramics: A diffuse interface approach, in: M. J. Hoffmann, G. Petzow (Eds.), Tailoring of Mechanical Properties of $\mathrm{Si}_{3} \mathrm{~N}_{4}$ Ceramics, Kluwer Academic Publishers, Amsterdam, 1994, pp. 291-301.

[98] M. F. Yan, R. M. Cannon, H. K. Bowen, Space charge, elastic field, and dipole contributions to equilibrium solute segregation at interfaces, Journal of Applied Physics 54 (1983) 764-778.

[99] C. M. Bishop, R. E. García, W. C. Carter, Effect of charge separation on the stability of large wavelength fluctuations during spinodal decomposition, Acta Materialia 51 (2003) 1517-1524.

[100] R. H. French, Origins and applications of London dispersion forces and Hamaker constants in ceramics, Journal of the American Ceramic Society 83 (2000) 2117-21146.

[101] W. H. Press, S. A. Teukolsky, W. T. Vetterling, B. P. Flannery, Numerical Recipes in C, Cambridge University Press, Cambridge, 1992. 
[102] A. A. Wheeler, W. J. Boettinger, G. B. McFadden, Phase-field model for isothermal phase transitions in binary alloys, Physical Review A 45 (1992) $7424-7439$.

[103] R. S. Qin, E. R. Wallach, A phase-field model coupled with a thermodynamic database, Acta Materialia 51 (2002) 6199-6210.

[104] H. Kobayashi, M. Ode, S. G. Kim, W. T. Kim, T. Suzuki, Phase-field model for solidification of ternary alloys coupled with thermodynamic database, Scripta Materialia 48 (2003) 699-694.

[105] S. H. Garofalini, W. Luo, Molecular dynamics simulations of calcium silicate intergranular films between silicon nitride crystals, Journal of the American Ceramic Society 86 (2003) 1741-1752.

[106] X. Su, S. H. Garofalini, Atomistic structure of calcium silicate intergranular films between prism and basal planes in silicon nitride: A molecular dynamics study, J. Mater. Res. 19 (2004) 752-758.

[107] G. S. Painter, P. F. Becher, W. A. Shelton, R. L. Satet, M. J. Hoffmann, First-principles study of rare-earth effects on grain growth and microstructure in beta- $\mathrm{Si}_{3} \mathrm{~N}_{4}$ ceramics, Physical Review B 70 (2004) 144108.

[108] D. Nguyen-Manh, D. J. H. Cockayne, M. Döoblinger, C. Marsh, A. P. Sutton, A. C. T. van Duin, Structural modelling of nano-amorphous Si-O-N films in silicon nitride ceramics, Electron Microscopy and Analysis 2003 Institute of Physics Conference Series 179 (2004) 139-142.

[109] C. M. Bishop, W. C. Carter, Relating atomistic grain boundary simulation results to the phase-field model, Computational Materials Science 25 (2002) $378-386$.

[110] J. Israelachvili, Intermolecular \& Surface Forces, Academic Press, London, 1992. 
[111] K. Johnston, M. W. Finnis, Electrostatic and entropic interactions between parallel interfaces separated by a glassy film, Journal of the American Ceramic Society 85 (2002) 2562-2568.

[112] J. Luo, Y.-M. Chiang, Equilibrium-thickness amorphous films on $11 \overline{2} 0$ surfaces of $\mathrm{Bi}_{2} \mathrm{O}_{3}$-doped $\mathrm{ZnO}$, Journal of European Ceramic Society 19 (1999) 697-701.

[113] J. Luo, Y.-M. Chiang, Existence and stability of nanometer-thick disordered films on oxide surfaces, Acta Mater. 48 (4501-4515) 2000.

[114] J. Luo, Y.-M. Chiang, R. M. Cannon, Nanometer-thick surficial films in oxide ceramics as a case of prewetting, submitted to Langmuir (2005). 
(a)
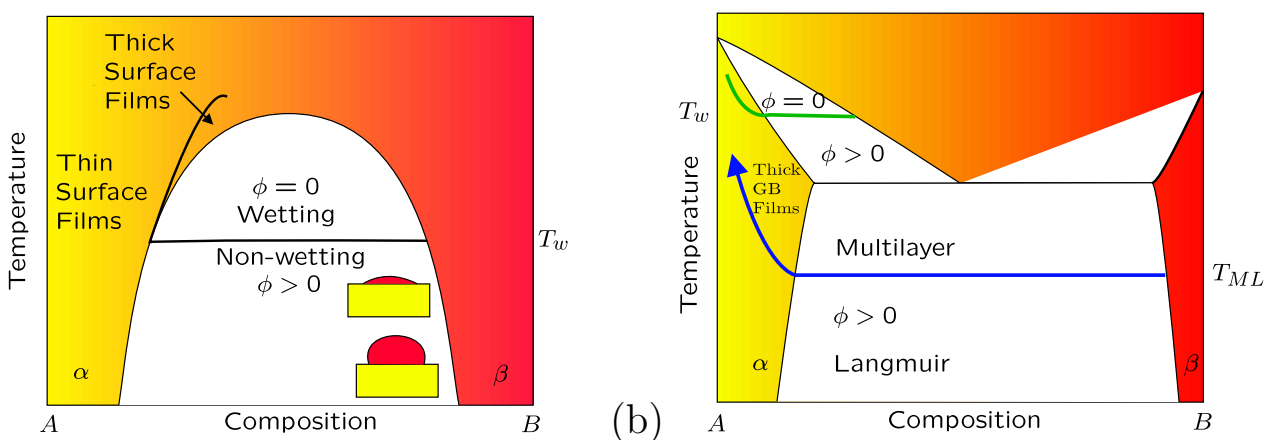

Fig. 1. Illustration of the parallel between critical wetting at surfaces and the prediction of grain boundary films. (a) emulates Cahn's original surficial phase diagram [20]. The two-phase region is separated into two regimes at the wetting temperature, $T_{W}$. At $T \geq T_{W}$, the $B$-rich phase completely wets the lower composition phase; at lower temperatures, the wetting angle, $\phi$, is non-zero. Cahn's critical wetting curve enters the single phase region at $T_{W}$ and it demarcates two different kinds of segregation behavior at the surface of the single phase. Those single phase compositions that lie closer to the completely wetting two-phase region will have surfaces with a thick, high-B composition layer. Traversing an isotherm towards $A$-rich compositions, the equilibrium layer will continuously reduce its thickness and composition until it reaches the critical wetting curve, where it will discontinuously reduce its equilibrium thickness and composition. This discontinuity reduces to zero at the critical wetting point. The thickness and the composition of the interface are equilibrium quantities. (b) illustrates potential similarities from the parallel model for grain boundaries that is reviewed in this paper. There are two two-phase regions in each of which there could be discontinuous changes in adsorption and wetting behavior. 

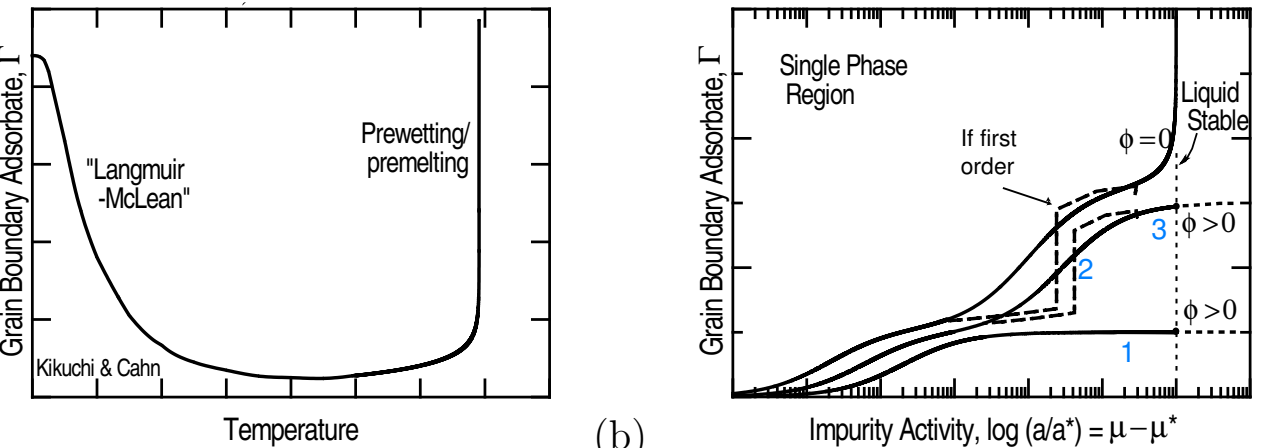

(a)

Impurity Activity, $\log \left(a / a^{*}\right)=\mu-\mu^{*}$

Fig. 2. Schematics of grain boundary adsorption isotherms. (a) the adsorption behavior predicted by Kikuchi and Cahn [27]. At low temperatures, there is a finite amount of adsorbate at specific low enthalpy sites. At higher temperatures approaching the bulk melting point, $\Gamma$ diverges as the grain boundary pre-melts to form a solute-enriched liquid with diverging thickness. (b) possible isotherms corresponding to different temperatures or misorientations as a function of chemical potential. The solid lines represent continuous transitions from monolayer adsorption to multilayer adsorption to pre-melting. The dashed lines indicate the possible locations of first order transitions from monolayer to multilayer adsorption.

(a)

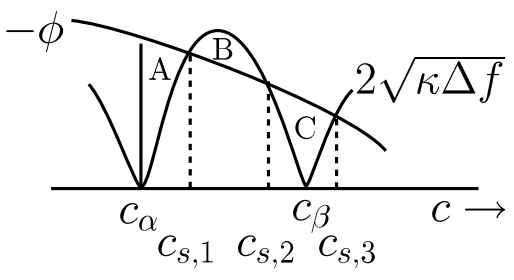

(b)

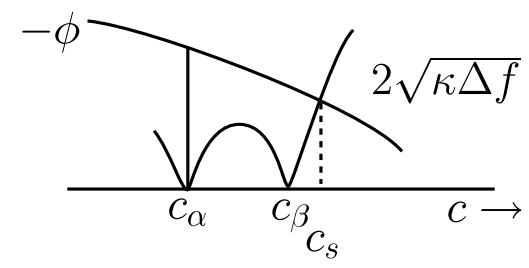

Fig. 3. Cahn's graphical construction for $c_{s}$ and equilibrium states when (a) $c_{0}=c_{\alpha}$ at $T_{1} \leq T_{w}$ and (b) $T_{2}>T_{w}$. Intersections of the two curves, $-\phi(c)$ and $2 \sqrt{\kappa \Delta f(c)}$, give values for $c_{s}$. At $T_{1}$, a comparison of the areas between the curves $(\mathrm{A}, \mathrm{B}$, and $\mathrm{C}$ corresponding to $c_{s, 1}, c_{s, 2}$, and $c_{s, 3}$ ) dictates whether the surface compositions correspond to stable, unstable or metastable solutions. At $T_{2}$, there is only one extremal $c_{s}$ which corresponds to perfect wetting. The behavior predicted here is plotted on the bulk phase diagram, Fig. 1(a). 


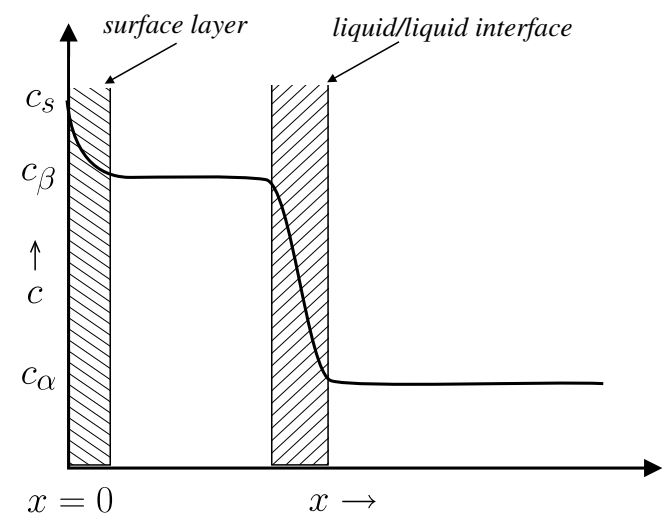

Fig. 4. Composition profile at $T>T_{w}$ and coexistence with $c_{0}=c_{\alpha}$ in a binary liquid system. The container wall is located at $x=0$ where the composition is B-rich at $c_{s}$. There is a wide layer of B-rich liquid then a transition to the bulk A-rich liquid.

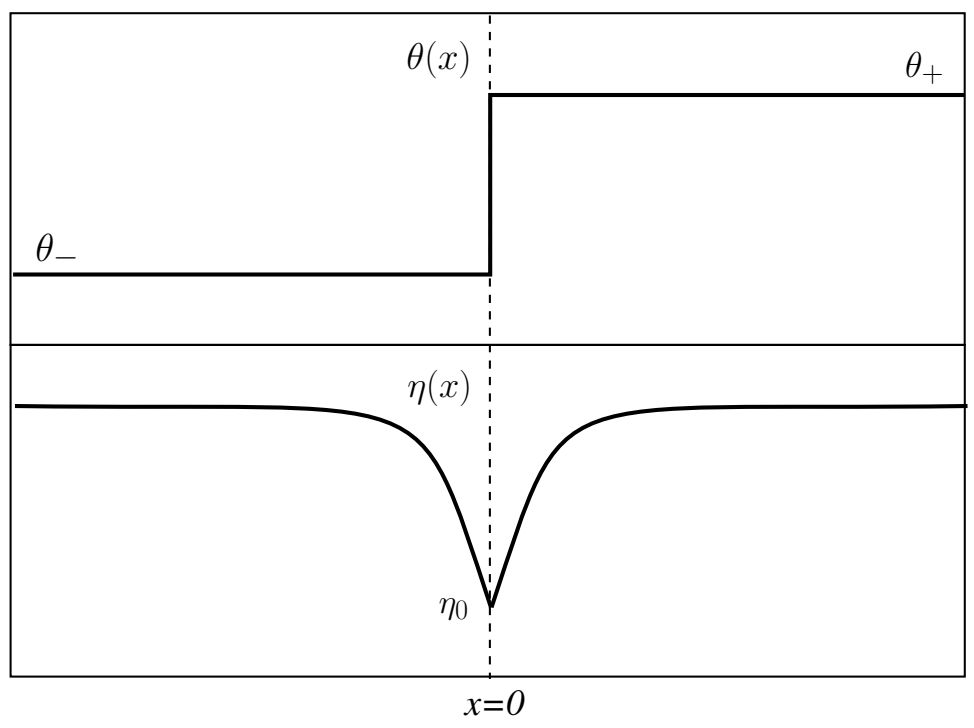

Fig. 5. Example profiles of structure, $\eta$, and orientation, $\theta$, across a planar grain boundary in the single-component KWC model with $h(\eta)=0$. The orientation is a step function located at $x=0$ and the core structure is described by $\eta_{0}$. 
(a)
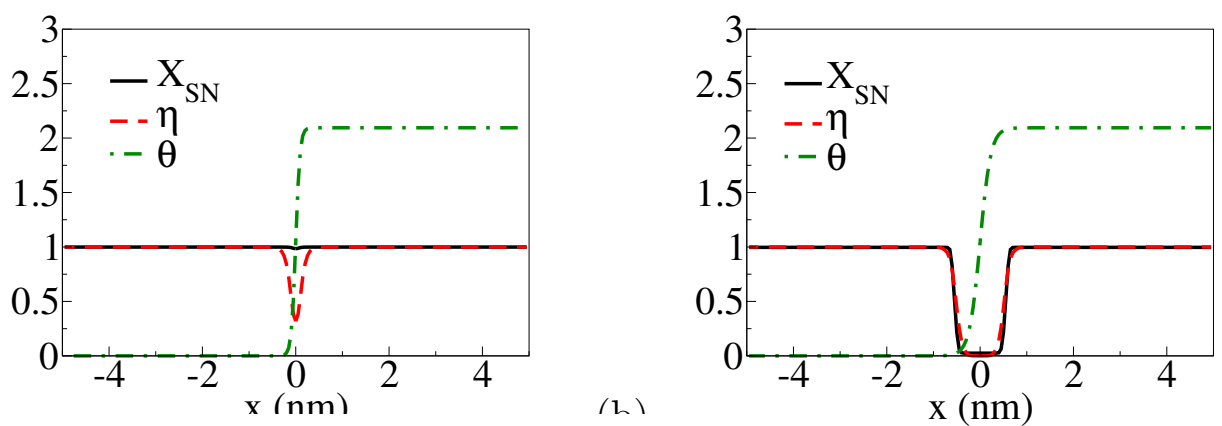

(h)

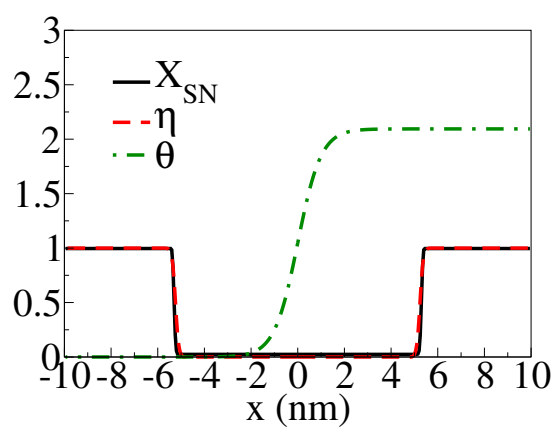

(c)

Fig. 6. Examples of grain boundaries varying from (a) ordered, low adsorption to (c) wide, disordered, high adsorption from simulations on silicon nitride from work in progress [78].

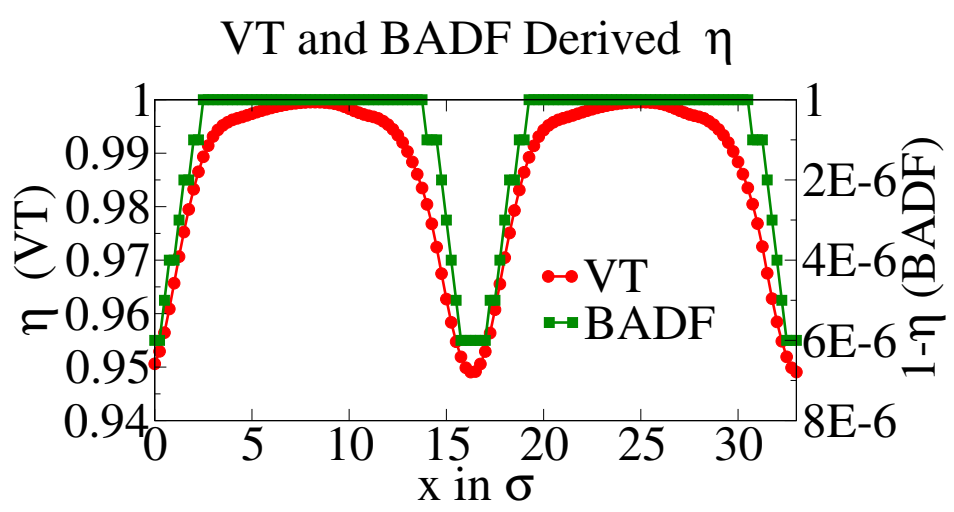

Fig. 7. A periodic cell containing two Si $\Sigma 5$ grain boundaries was relaxed at $0 \mathrm{~K}$. Coarse-grained measures of $\eta$ as a function of position in units of $\sigma$ were calculated using the BADF (green square) and VT (red circle) methods [109,77]. 